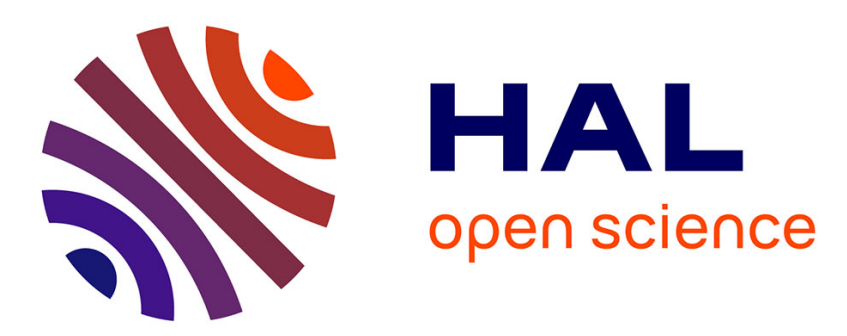

\title{
Novel concept of interferometric control of the moving coil in watt balance
}

M Wakim, L Chassagne, S Topsu, Y Alayli, Jp. Wallerand, Patrick Juncar, G Geneves

\section{- To cite this version:}

M Wakim, L Chassagne, S Topsu, Y Alayli, Jp. Wallerand, et al.. Novel concept of interferometric control of the moving coil in watt balance. SPIE Conference on International Congress on Optics and Optoelectronics, Apr 2007, Prague, Czech Republic. hal-01212820

\section{HAL Id: hal-01212820 \\ https://hal.science/hal-01212820}

Submitted on 7 Oct 2015

HAL is a multi-disciplinary open access archive for the deposit and dissemination of scientific research documents, whether they are published or not. The documents may come from teaching and research institutions in France or abroad, or from public or private research centers.
L'archive ouverte pluridisciplinaire HAL, est destinée au dépôt et à la diffusion de documents scientifiques de niveau recherche, publiés ou non, émanant des établissements d'enseignement et de recherche français ou étrangers, des laboratoires publics ou privés. 


\title{
Novel concept of interferometric control of the moving coil in watt balance
}

\author{
M. Wakim ${ }^{\text {a,c,* }}$, L. Chassagne ${ }^{a}$, S. Topsu ${ }^{a}$, Y. Alayli ${ }^{\mathrm{a}}$, J.P.Wallerand ${ }^{\mathrm{b}}$, P. Juncar ${ }^{\mathrm{b}}$ and G.Geneves ${ }^{\mathrm{c}}$ \\ ${ }^{a}$ LIS Versailles, Department of physics, University of Versailles, 45 Avenue des Etats-Unis, 78035 \\ Versailles, France \\ ${ }^{\mathrm{b}}$ LNE-INM, 292 rue Saint-Martin, 75013 Paris, France \\ ${ }^{c}$ LNE, 29, avenue Roger Hennequin 78197 Trappes, France
}

\begin{abstract}
One of the most promising projects for the new definition of the kilogram is the watt balance. It uses a two-mode procedure: static mode and dynamic mode. One of the key points is to control the velocity, the yaw and the pitch of a moving coil during the dynamic mode. We have developed a specific method based on the use of a homemade double pass heterodyne interferometer, a two-level translation stage, and a home-made high frequency phase shifting electronic circuit. However, the commercial heterodyne sensor is limited in power and has polarization limitations. In this paper, we propose a novel and specific heterodyne interferometer able to insure all previous demands to control the displacements of the moving coil in order to reduce the velocity noise. The laser source is powerful enough to ensure beams of measurements, the laser is frequency stabilized at 532 iodine atomic transition to ensure traceability, and all the polarization defaults of the classical heterodyne interferometer have been minimized in order to minimize non-linearity effects. The most original point is that the reference beam is lock-in on an external electronic reference, which allows to be less sensitive to vibration and polarization defects of optical components.
\end{abstract}

Keywords: watt balance, kilogram, precision motion control, nanotechnology, heterodyne laser interferometer.

\section{INTRODUCTION}

The kilogram is the last remaining unit of the International System of Units which is still defined by a material artefact. This artefact is made from an alloy of $90 \%$ platinum, $10 \%$ iridium. In spite of the good choice of the alloy, Surface contamination and bulk outgassing phenomena can vary the amount of matter in the artefact. At the end of the twentieth century international reference and all national references were compared between them. Variations of $3.10^{-8}$ appeared between the international reference and the mean value of all national prototypes [1]. Since it is very difficult to know which mass has varied or if all masses have varied, actual reference is maintained till finding a new reference. Several laboratories of metrology develop methods to link the unit of mass to fundamental or atomic constants hoping to find a new definition of the kilogram. One of the most promising methods seems to be the watt balance. The"Laboratoire National de métrologie et d'Essais" (LNE) is developing its own watt balance [2]. The principle was proposed by B. P. Kibble (NPL) in 1975 [3]. The aim of the project is to compare a mechanical power with an electromagnetic power in order to link mass to the Planck's constant [4-6]. The measurement system is mainly composed of a force comparator, a coil immersed in a $1 \mathrm{~T}$ radial induction field suspended below one of its arms. The coil, weighing $2 \mathrm{~kg}$, is made from a $260 \mathrm{~mm}$ diameter epoxy cylindrical support on which 600 spires are enrolled. The watt balance uses two-mode procedure [7]: static mode and dynamic mode. The Laplace's force counterbalances the mass of the etalon in the static mode. It's generated when the coil is supplied by a known current. In the dynamic mode the coil moves vertically in the permanent magnetic field with a velocity of $2 \mathrm{~mm} / \mathrm{s}$ and induces Electromotive force $(\varepsilon)$ of $1 \mathrm{~V}$. The vertical displacement is insured thanks to a set of six flexure strips. By combining the two-mode results and measuring the electrical quantities using the Josephson Effect [8] and the quantum hall effect [9], mass can be expressed as follows:

* Marwan.wakim@lne.fr phone: 0033160392194, fax: 0033130162841

Web site : www.lne.fr 


$$
m=K \frac{f_{J}^{2}}{g \cdot V} h
$$

Where $K$ a constant, $f_{J}$ is the Josephson frequency, $g$ the local acceleration of gravity, $V$ the velocity of the moving-coil, and $h$ the Planck's constant. To obtain " $m$ " with an uncertainty of $10^{-8}$, all physical parameters of the above formula should be measured and controlled with an uncertainty better than $10^{-8}$ and notably the velocity $(V)$.

We propose in this paper a novel method to measure and control velocity dedicated to the French watt balance. The method is based on the use of a heterodyne Michelson's interferometer, a two level translation stage, and a home-made high frequency phase shifting electronic circuit. Our method allows a centimetric displacement with an intrinsic relative accuracy on the velocity of $1.9 \times 10^{-9}$ over $100 \mathrm{~s}$.

\section{PRINCIPLE AND EXPERIMETAL SETUP}

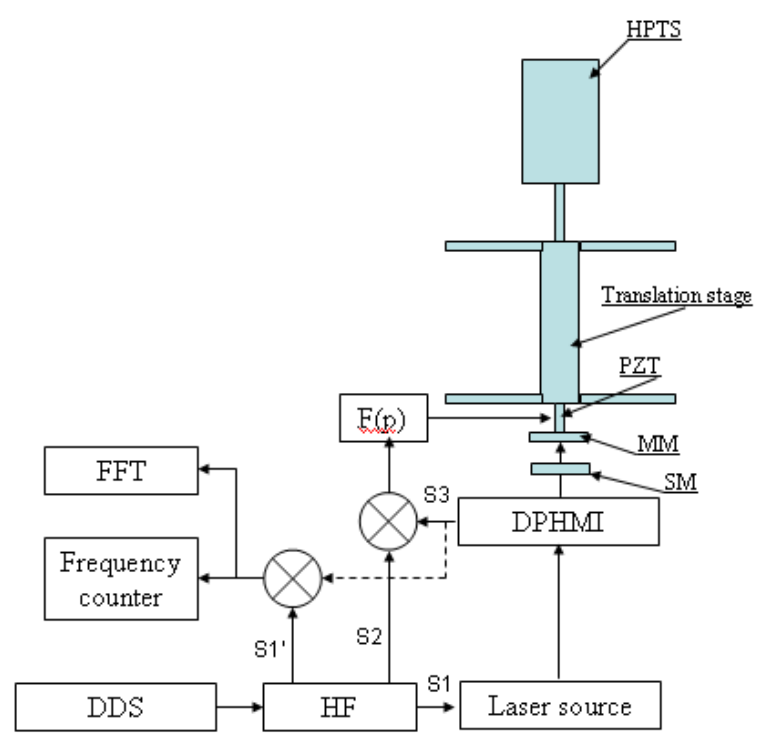

Fig: 1. experimental setup. DDS, digital direct signal generator; F(p), proportional integral derivative system; HF, high frequency electronic circuit, MM, movable mirror, SM stationary mirror. FFT, fast Fourier transform apparatus; HPTS, high precision translation stage; DPHMI, double pass heterodyne Michelson's interferometer; PZT piezoelectric actuator;

Linear translation stage displaces the coil in the magnetic field with a velocity of $2 \mathrm{~mm} / \mathrm{s}$. Even with the best high precision translation stages, it is impossible to obtain a stability of the velocity below to $10^{-6}$ in relative value over centimetric displacement. In the other hand Constraints of the experience force the use of a motor with stability on the velocity of $10^{-4}$ (Motor is used in vertical and in a vacuum enclosure). Moreover vertical movement of the coil must be perfectly vertical, horizontal component of the velocity induces an undesired electromotive force and disturb the electrical measurements. For this purpose an intermediate vertical translation stage was developed in the interest to guide the coil according to the vertical direction. The guide stage is decoupled of the linear translation stage and insures straightness within a few micrometers over $80 \mathrm{~mm}$ of travel. Moving the coil on an $80 \mathrm{~mm}$ of travel with a relative uncertainty of $10^{-9}$ requests a second stage. Our method is based on the use of a double pass heterodyne Michelson's interferometer, a two-level translation stage, and a homemade high frequency phase-shifting electronic circuit (fig.1). The two level translation stage is made by superposing a linear motor and a piezoelectric actuator. The piezoelectric actuator, placed between the translation stage and the coil, corrects the defect of the first stage. The coil is replaced by a simple mirror that consist the movable mirror of the interferometer. Commercial laser head generates two orthogonal polarized beams with $20 \mathrm{MHz}$ of difference between frequencies. The first beam constitutes the measurement signal (reflect on MM) and the second one constitutes the reference signal (reflect on SM). Information on the position and the velocity is coded in the output signal of the interferometer. Wavelength of the Laser head is carefully chosen to have a traceable measurement with regard of the meter and the second definition. For this purpose we choose one of the 
wavelengths recommended by the "comite international des poids et des mesures". The home made high frequency electronic circuit generates three signals s1, s'1, and s2 at the same frequency [10-11]. The first one (S1) feeds the Bragg cell and creates a synchronous heterodyning with the electronic signals. Signals (S'1, S2) are phase compared to the signal (S3) at the exit of the interferometer. Error signal resulting of the mixing of S2 and S3 creates the input of the proportional integrator derivative module that controls the piezoelectric actuator length. In static, imagine that vibration on the system moves the mirror away or closer to the reference mirror. On the mixer level appears a non null signal error due to the Doppler Effect. Doppler Effect results in a positive or negative phase shift by an amount of $2 \pi$ for a $\lambda / 4$ of displacement, neglecting refractive index of the air. The displacement can be deduced according with the formula:

$$
\Delta x=\frac{\lambda}{4} \times \frac{\Delta \phi}{2 \pi}
$$

Where $\lambda$ is the wave length of the measurement beam and $\Delta \Phi$ the phase shift. When the position of the mirror is controlled, the noise level is about $1 \mathrm{~nm}$ of amplitude. Let us handle the problem at reverse. Suppose that S1 or S2 undergo a phase shift. Here again error $\varepsilon$ becomes non null and the mirror moves to compensate the phase shift imposed by the electronic circuit. Mirror can move in the 2 ways of the vertical direction depending on where the phase shifts are imposed with a step whose value is defined by the angle of the phase shift. Electronic circuit is able to impose phase shifts up to $2 \pi / 32(5 \mathrm{~nm})$ in normal mode and $2 \pi / 612$ in differential mode (260pm) [12]. Differential mode consists to impose phase shifts in the same time on the two signals (S1 and S2) Knowing that the resolution of our interferometer doesn't exceed $0.31 \mathrm{~nm}$ so we work in normal mode to control the velocity with steps of $5 \mathrm{~nm}$ which is sufficient to get $10^{-9}$ of relative stability on the velocity. When the movable mirror moves at $2 \mathrm{~mm} / \mathrm{s}$ the phase shift becomes too high and shifts the value of the frequency at the exit of the interferometer. Signal demodulated with a reference signal s'1 to reconstitute the Doppler frequency $(12,64 \mathrm{khz}$ for $2 \mathrm{~mm} / \mathrm{s}$ of velocity). By imposing the same rhythm of phase shift on $\mathrm{S} 1$ or S2 (depends on the direction) and by controlling with the servo loop the piezoelectric will change the real time position of the mirror in relation with the motor referential and compensate defects of the motor velocity.

\section{RESULTS AND DISCUSSIONS}

The optical signal at the exit of the interferometer is demodulated to obtain the Doppler frequency that contains the information of the velocity and its stability. The resulting signal is sent into a FFT apparatus (SR785, Stanford) and a high stability frequency counter HP53132A with a relative accuracy of $10^{-10}$. The first apparatus monitors the control quality by examining the peak width. The second one measures the frequency and collects the real time value in a computer. The software uses a non classical statistic method to plot the Allan standard deviation curve [13 ]. This method is a graphical data analysis technique used like a metrological tool to make an estimation of the stability of primary frequency standards [9].

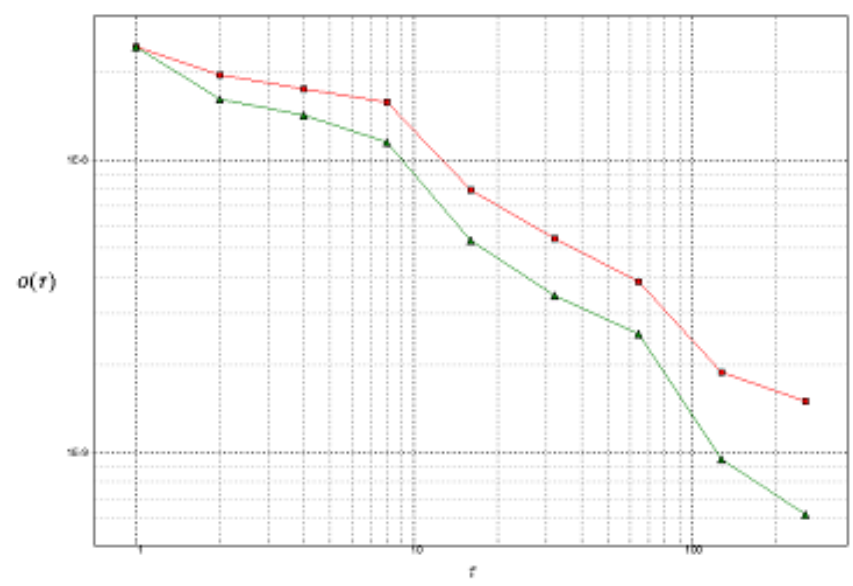

Fig.2: Allan standard deviation of the Doppler frequency shift for a velocity of $2 \mathrm{~mm} / \mathrm{s}$ in the vertical direction. Each point is averaged over 1 second. 
The mirror moves on an $80 \mathrm{~mm}$ travel at $2 \mathrm{~mm} / \mathrm{s}$ of velocity corresponding to 40 seconds by travel. We consider that the motor and the translation system take 10 seconds to achieve the transient state (acceleration, deceleration and vibration) at the beginning and the end of the travel leading 20 seconds of acquisition time in steady state. In this last state a measure of the Doppler frequency shift is took by averaging measurements on each second. 20 points are recorded by travel. Step is repeated 100 times to get 2000 points. Figure 2 represents a standard deviation of the Doppler frequency shift corresponding to a velocity of the moving mirror of $2 \mathrm{~mm} / \mathrm{s}$ in the vertical direction. The results show that a stability of $2 \times 10^{-8}$ is occurred over 1 second. The same figure makes an estimation on stability of the velocity and shows a stability of $1.9 \times 10^{-9}$ over 100 seconds. Results are obtained in an air conditioned room where temperature is controlled to 0.1 degrees Celsius. The watt balance experiment is planned to be placed under vacuum, which will ameliorate results.

\section{ALTERNATIVE METHOD}

In spite of good results acquired with the system described above some strength can be harmful to the good functioning of the watt balance. Since the horizontal component of the velocity annoys the measurement of the Josephson Effect it's interesting to measure the pitch and yaw of the coil. Controlling velocity and measuring simultaneously pitch and yaw demands a system able to work on three channels. Thus the laser beam will be divided into three beams. The small power of the laser head used in this method (500microwatt) will be a limitation. The laser source contains two orthogonal linear polarized beams with different frequencies. Practically polarisations present some elipticity and 0.5 degrees of uncertainty on the perpendicularity. Knowing that the watt balance will be installed in a vacuum enclosure, polarizations risk to be lost by the changing of the refractive index of air and by the crossing of the optical window. Using fiber optic to carry the signal in the vacuum enclosure is not the best solution, Polarizations risk to be mixed in the fiber. Moreover laser head can not be installed in the vacuum enclosure because of enormous thermal dissipation.

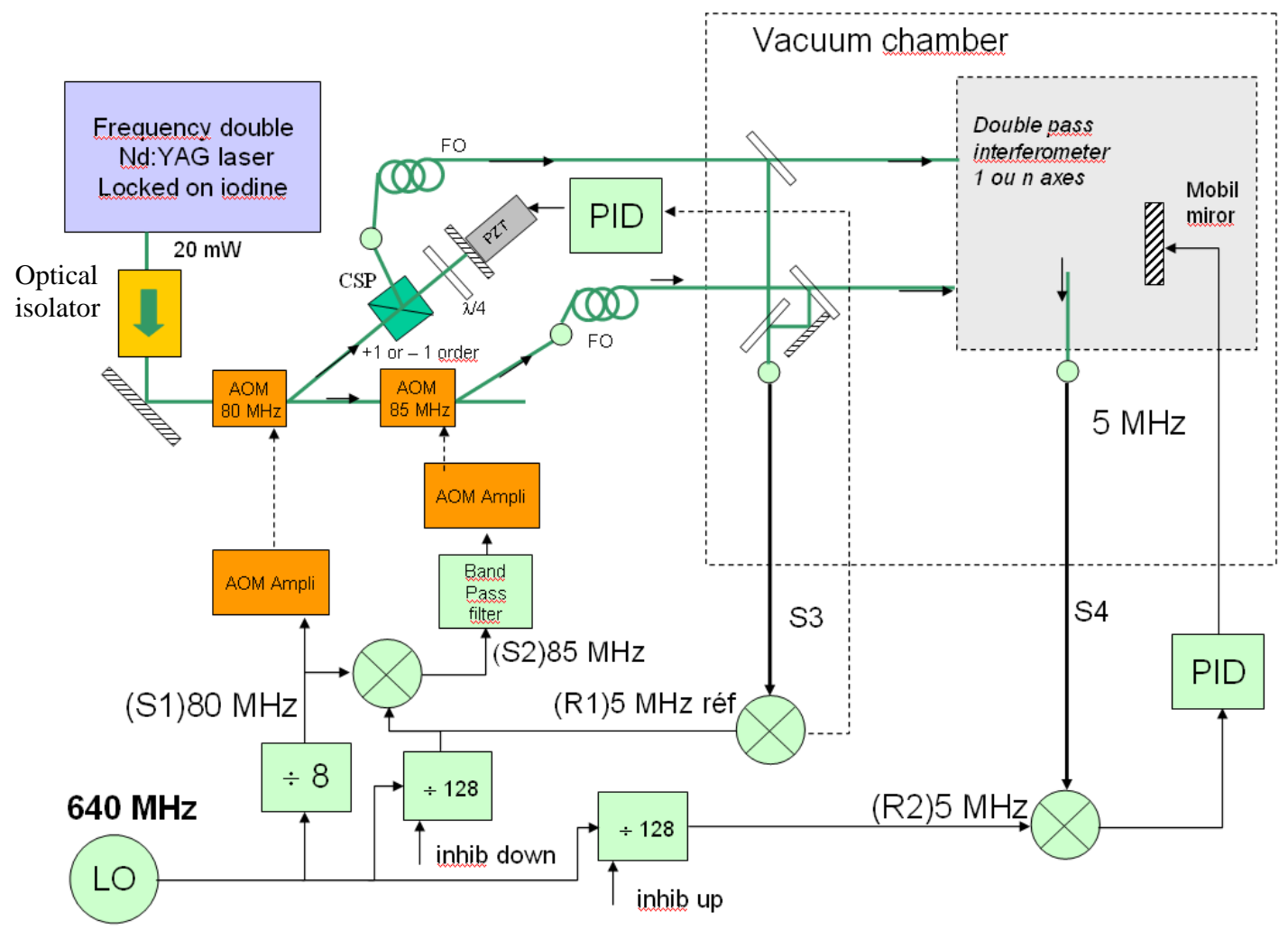

Fig.3: OL, local oscillator at $640 \mathrm{MHz} ; \div 8, \div 128$, very low phase noise synthesiser divider; AOM, acousto-optic modulator; AOM Ampli, electronic amplifier for the signal feeds the acousto-optic modulator; PZT piezoelectric actuator; PID, proportional integrator derivative module; FO, fiber optic cable. 
In order to resolve those problems a new concept for a new heterodyne laser source is setup in order to adapt the method to the watt balance (fig. 3). It's very important to find a system that insures: power, linearity, good resolution, small thermal dissipation, traceability and to be fibered. Nowadays no commercial system is able to insure those criteria. We have developed an alternative method able to answer to all our demands. The principle is based on the use of a Nd:yag doubled laser with an accordable wavelength that generates a monomode longitudinal laser beam on $532 \mathrm{~nm}$ with $20 \mathrm{mw}$ of power. Faraday isolator is used to prevent unwanted feedback to the laser and made the optical oscillator more stable. $1 \mathrm{mw}$ of the total power is used to lock on laser frequency to a saturation absorption line of the iodine hyperfine structure transition. Locking the laser frequency on the iodine absorption peak permit stability on the frequency of $10^{-12}$. To reduce the absorption of the laser beam in the iodine cell and to have the best frequency stability, the temperature of the cold finger is fixed on -15 degree Celsius in order to reduce pressure to $0.8 \mathrm{~Pa}$ in the cell thanks to Peltier Effect Thermoelectric Cooler. The other part of the laser beam passes trough a two acousto-optic modulators. The two first order beams are injected into two optical fibers. Frequencies of the two first order beams at the exit of the acoustooptical modulators are shifted of the value of the frequency that supplies it. The two acousto-optical modulators are fed respectively with two electrical signals $\mathrm{S} 1$ and $\mathrm{S} 2$ with $80 \mathrm{MHz}$ and $85 \mathrm{MHz}$ of frequency. The two first order beams generated from the two acousto-optical modulators with a difference of frequency of $5 \mathrm{MHz}$ are injected in two optical fibers to bring signals into the vacuum enclosure. The first signal is directly injected. The second one passes through a polarizing beam splitter (PBS) and a quarter waveplate before being reflected by the mirror (M). After the passing in the quarter waveplate $(\lambda / 4)$ the polarization is converted from horizontally linear to right circular polarization. Reflected beam with left circular polarization pass again in the same quarter waveplate $(\lambda / 4)$ to obtain a vertically linear polarization and reflects on the polarizing beam splitter (PBS) and than it will be injected in a fiber optic. The mirror is fixed on a piezoelectric actuator (PZT). The system is conceived to lock on the phase at the input of the vacuum enclosure. A home made high frequency electronic card generates two signals at $80 \mathrm{MHz}$ and $85 \mathrm{MHz}$ by dividing the signal of the local oscillator OL (640MHz) by 8 and 128 thanks to a very low phase noise synthesiser divider. The signal at $80 \mathrm{MHz}(\mathrm{S} 1)$ supplies the first acousto-optical modulator. The other one (R1) is mixed with the signal (S1) at $80 \mathrm{MHz}$. A band-pass filter isolates all frequencies and passes the signal (S2) at $85 \mathrm{MHz}$ that supplies the second acousto-optical modulator. Another very low phase noise synthesiser divider divides the signal of the local oscillator to obtain the reference signal R2.

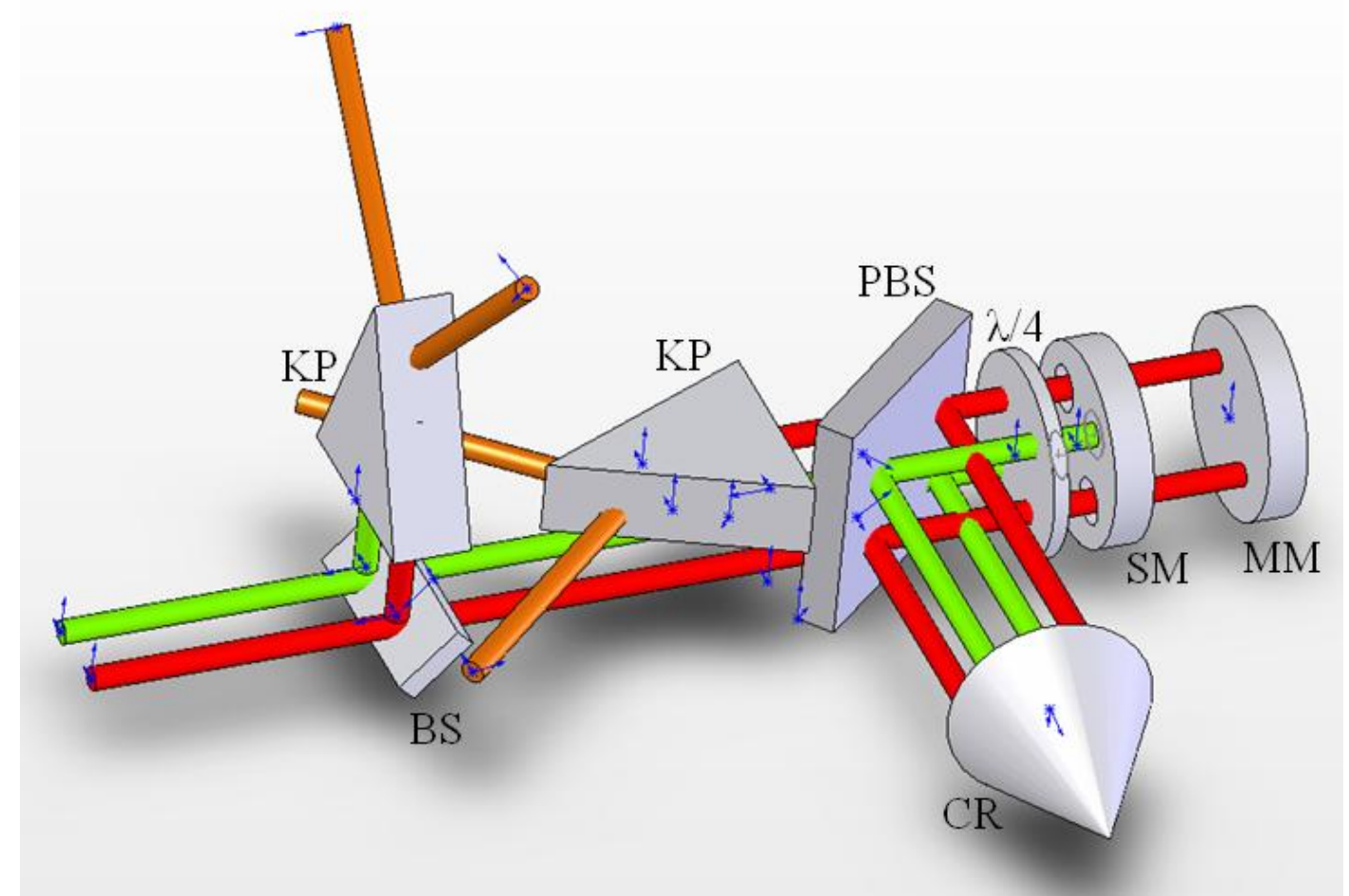

Fig.4: BS: beam splitter, KP: Kösters Interference double prism; PBS, polarizing beam splitter; CR, cone reflector; $\lambda / 4$, quarter waveplate; SM, stationary mirror; MM movable mirror. 
In the vacuum enclosure the two beams are divided by a beam-splitter (fig.4). The first two beams are beating together thanks to a Kösters Interference double prism and are phase compared to electronic signal (S1) at $5 \mathrm{MHz}$. The phase of the beating signal is locked on the electronic signal (R1) via an analogical servo-loop. The servo-loop acts on the piezoelectric actuator to move mirror and change the length of the optical path and than to fix the beating frequency at 5 MHz. system allowing to overcome all frequency changing due to thermal and all other effects in fibers. The other part of the two beams beats at the exit of a homemade double pass heterodyne interferometer. The interferometer is composed from a polarizing beam splitter (PBS) a quarter waveplate $(\lambda / 4)$ and a cone reflector (CR). The beams follow the path like showed in the figure 4 . The first beam constitutes the reference beam and is reflected on the holed mirror (SM). The second one constitutes the measurement beam; it passes through the holed mirror and is reflected on the movable mirror (MM). The two beams are beating together again in a Kösters Interference double prism and are phase compared to the electronic signal (R2) at $5 \mathrm{MHz}$ and another analog servo-loop lock-in the phase consequently the position of the movable mirror. As seen previously phase shifts can be imposed on the signal R1 or R2 to move the movable mirror in two directions but this time the resolution is better thanks to a smaller wavelength and difference between the two frequencies.

$$
\Delta p=\frac{\lambda}{4 \times 128}=1.039 \mathrm{~nm}
$$

Where $\Delta p$ the minimum is the displacement achieved in normal mode and $\lambda$ the wavelength of the laser beam.

\section{CONCLUSION}

We have developed a new method to measure and control the velocity of the moving coil in the watt balance with a relative uncertainty of $1.9 \times 10^{-9}$ over 100 seconds. The developed system achieved satisfactory results, however it's not enough adapted to the watt balance. In this perspective a new method is conceived to overcome drawbacks and eliminates weakness points. The system was conceived to be fibered insuring good resolution, high power to measure simultaneously velocity, pitch and yaw. Moreover the system insures linearity, small thermal dissipation, and traceability. The new system will be placed under vacuum; we expect better results and more stability on the velocity. The high power generated by our system will allow in the future the control of the velocity of three lateral points of the coil and thus enables us to surpass the pitch and yaw effects.

\section{REFERENCES}

[1] G. GIRARD "The Third Periodic Verification of National Prototypes of the Kilogram (1988-1992)" Metrologia, 31, 317-336, 1994

[2] GENEVES G. ; GOURNAY P. ; GOSSET A. ; LECOLLINET M. ; VILLAR F. ; PINOT P. ; JUNCAR P. ; CLAIRON A. ; LANDRAGIN A. ; HOLLEVILLE D. ; PEREIRA DOS SANTOS F. ; DAVID J. ; BESBES M. ; ALVES Francisco ; CHASSAGNE L. ; TOPCU S. , “ The BNM Watt Balance Project “, IEEE Trans. Instrum. Meas., 54, 850-853, 2005.

[3] B.P. Kibble, “Atomic Masses and Fundamental Constants”, edited by J. H. Sanders and A. H. Wapstra (Plenum, New York, 5, 545, 1976.

[4] Y. Fujii, Y. Miki, F. Shiota, and T. Morokuma, IEEE Trans. Instrum. Meas. 50, 5802001.

[5] B. P. Kibble, I. A. Robinson, and J. H. Bellis, Metrologia 27, 1731990.

[6] V. Bego, J. Butorac, and D. Illié, IEEE Trans. Instrum. Meas. 48, 212 (1999).

[7] A. Eichenberger, B. Jeckelmann, P. Richard, "Tracing the Planck's constant to the kilogram by electromechanical methods ", Metrologia, 40, 356-365, 2003.

[8] C. A. Hamilton and Y. H. Tang, "Evaluating the uncertainty of Josephson voltage standards" Metrologia, 36, 531999.

[9] B. JECKELMANN and B. JEANNERET, "The quantum Hall effect as an electrical resistance standard" Rep. Prog. Phys., 64 1603-1655, 2001. 
[10] DPMI Accessory Operating Manuel OMP-0223E, Zygo, 2002.

[11] S. Topçu, L. Chassagne, D. Haddad, Y. Alayli, Patrick Juncar, High accuracy velocity control method for the French moving coil watt balance, Review of Scientific Instruments, 75, N¹1, 4824-4827, 2004.

[12] Topcu S., Chassagne L., Haddad D., Juncar P. et Alayli Y., « Heterodyne interferometric technique for displacement control at the nanometric scale », Rev. Sci. Instr., 74, 4876-4880, 2003.

[13] L. Chassagne, S. Topcu, Y. Alayli, P. Juncar, "Highly accurate positioning control method for piezoelectric actuators based on phase-shifting optoelectronics", Meas. Sci. Technol., 16, No 9, 1771-1777,2005

[14] Allan D. W., «Time and Frequency (Time-Domain) Characterization, Estimation, and Prediction of Precision Clocks and Oscillators», IEEE Transactions on Ultrasonics, Ferroelectrics and Frequency Control, 34, 647654, 1987. 